\title{
Rehabilitating rimonabant
}

\author{
Friedrich C. Luft
}

Published online: 17 April 2013

(C) Springer-Verlag Berlin Heidelberg 2013

The two cannabinoid receptor subtypes (CB1 and CB2) belong to the $G$ protein-coupled receptor superfamily. The CB1 receptor is primarily expressed in the central nervous system but also resides in the lungs, liver, and kidneys. The CB2 receptor is heavily expressed on immune and hematopoietic cells [1]. Additional "novel" cannabinoid receptors may exist on endothelial cells [2]. The amino acid sequences of the receptors are about $44 \%$ similar, although the seven transmembrane-spanning segments exhibit a higher homology. The receptor ligands fall into three major categories, endocannabinoids produced by the body, plant cannabinoids like tetrahydrocannabinol (THC), and synthetic cannabinoids. The affinity of an individual cannabinoid to each receptor subtype determines the effect of that cannabinoid.

CB1 receptors are responsible for endocannabinoid-mediated depolarization-induced suppression of inhibition. In this plastic process, depolarization of a single neuron induces a reduction in GABA-mediated neurotransmission. Endocannabinoids released from the depolarized postsynaptic neuron bind to CB1 receptors on the presynaptic neuron and cause a reduction in GABA release. CB1 receptor gene-deleted mice have a shortened lifespan, impaired locomotor activity, and hypoalgesia [3]. They also do not respond to THC. CB2 receptors mediate effects of endocannabinoids or synthetic agonists in immune-derived cells including various populations of $\mathrm{T}$ and $\mathrm{B}$ lymphocytes, monocytes/macrophages, dendritic cells, mast cells, microglia in the brain, and Kupffer cells in the liver [4]. Also in their repertoire are other potential cellular targets such as endothelial and smooth muscle cells, fibroblasts in various organs, cardiomyocytes, and certain neuronal elements of the peripheral or central nervous systems.

F. C. Luft $(\bowtie)$

Experimental and Clinical Research Center, and Max-Delbrück

Center, Lindenbergerweg

80, 13125 Berlin, Germany

e-mail: luft@charite.de
As already mentioned [2], additional cannabinoid receptors may exist as suggested by actions of cannabidiol that produces cannabinoid-like effects on blood pressure and inflammation, yet does not activate either CB1 or CB2 receptors $[5,6]$. The $N$-arrachidonoyl glycine receptor GPR18 and the orphan receptor GPR55 are also candidates, since they respond to various endogenous and exogenous cannabinoid ligands. Cannabinoid receptors signal by the production of the second messenger molecule cyclic AMP. However, they also engage other pathways. Implicated are potassium ion channels, calcium channels, protein kinase A and C, Raf-1, ERK, JNK, p38, c-fos, c-jun, and many more signaling molecules [7].

Rimonabant is an inverse agonist (inhibitor) for the CB1 receptor [8]. The drug reduces appetite and is of value in persons seeking to quit smoking. Rimonabant also reduced resumption of cocaine-seeking responses and may also reduce ethanol and opiate-seeking behavior. Interestingly, rimonabant also blocked the cardiovascular effects of THC and reduced heart rate in subjects taking THC by almost $60 \%$ [9]. The drug began a promising and progressing career in patients with obesity and metabolic syndrome. The patients lost weight, and their cardiovascular risk factors were substantially reduced. This drug appeared to be a compound that "had it all." Presumably through actions on the CB1 receptor, rimonabant was associated with depression and suicidal ideation, and further clinical development of the compound has been abandoned.

In this issue, Slavic et al. [10] report on the effect of CB1 inhibition on cardiac function and remodeling after experimental myocardial infarction and after experimental metabolic syndrome. They inspected two different rat models. In the first, the left anterior coronary artery was ligated to induce myocardial infarction. Rimonabant had been administered in one group 7 days in advance and continued for 
6 weeks thereafter. In another group, treatment was begun after the myocardial infarction was produced and continued for 6 weeks. Although acute mortality was not influenced, CB1 pretreatment blockade resulted in various improvements, including improved ventricular function, remodeling characteristics, less fibrosis, lower brain-natriuretic peptide levels, and relative downregulation of transforming growth factor- $\beta$ (TGF- $\beta$ ). The latter was documented in cultured fibroblasts where rimonabant also downregulated TGF- $\beta$ as well as matrix-metalloproteinase-9. Furthermore, the improvements were extended to the entire vascular tree, since aortic stiffness was reduced by rimonabant. The results are all the more impressive since many of the findings were based on direct invasive catheter measurements. Rimonabant also improved ventricular function and led to weight loss in the obese spontaneously hypertensive rat model of the metabolic syndrome in independent studies. The current findings are not entirely new, since others have reported cardioprotective properties of rimonabant in earlier studies [11].

How can we interpret these findings in light of what we already know about cannabinoid receptors and the functions of rimonabant? Montecucco and DiMarzo have extensively reviewed the endocannabinoid system in cardiovascular physiology and disease [12]. Cardiac and vascular tissue seems to be well outfitted with endocannabinoid receptors, including CB1, CB2, the transient receptor-potential channel TRPV1, and participating molecules. The data presented by Slavic et al. [10] imply that blocking CB1 receptors led to the beneficial effects in their model. On the other hand, CB2 receptor activation was found to ameliorate ischemia reperfusion injury in the myocardium. Unclear is possible participation of GPR18 and GPR55 in the responses Slavic et al. observed [10]. Whether or not blockade of the $\mathrm{CB} 1$ receptor would have led to increased occupancy of other receptors is unknown. An interesting positive control in the current studies could have been inclusion of a group receiving a $\mathrm{CB} 1$ agonist, rather than antagonist. Studies on THC in CB1 and CB2 genedeleted mice indicated that the hypotension caused by THC, which is observed clinically, involved direct activation of CB1 receptor. Participation of endocannabinoids in the responses by mechanisms relying on other receptors is also unclear. Anandamide apparently addresses CB1 receptors in the central nervous system, but CB2 receptors peripherally. Cannabidiol, which has little affinity for $\mathrm{CB} 1$ or $\mathrm{CB} 2$ receptors but instead which may work via TRPV1 or other mechanisms, was found to attenuate cardiac dysfunction, oxidative stress, fibrosis, inflammation, and death signaling pathways in a model of cardiomyopathy [13]. The complexity of the

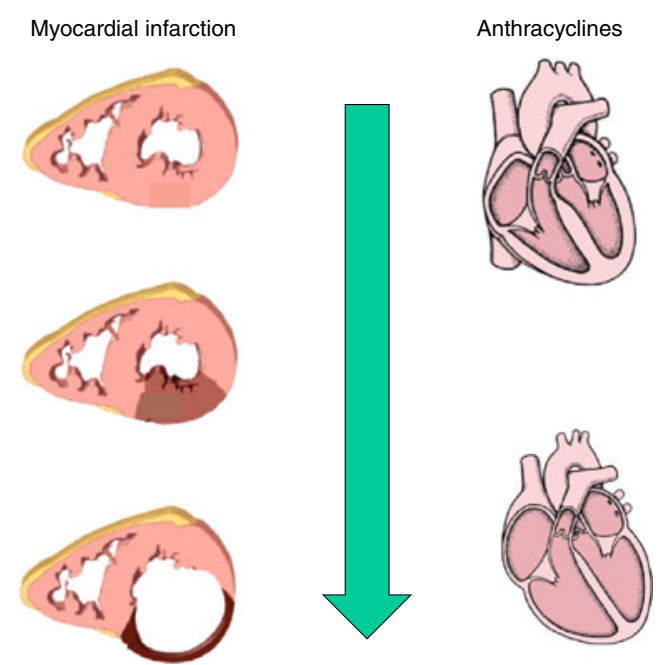

Inhibition of CB1 and activation of CB2?

Fig. 1 Myocardial infarction leads to unfavorable remodeling and heart failure. Anthracyclines lead to dilative cardiomyopathy. Could $\mathrm{CB} 1$ inhibition or $\mathrm{CB} 2$ activation ameliorate this state of affairs?

endocannabinoid system would require a series of conditional gene deletions in various tissues to dissect out the detailed mechanisms of the authors' findings.

Are there possibilities that the authors' findings could be translated into any clinical utility? As a prophylactic treatment for the metabolic syndrome or for the consequences of myocardial infarction, rimonabant would no longer appear to be a likely option. The debacle following withdrawal of rimonabant from clinical use would tend to make manufacturers very wary of introducing CB1 receptor blockers into the clinical arena, although "second generation" compounds with less CNS activity are said to be under consideration. However, there are orphan heart diseases for which we currently have little in the way of prophylaxis to offer. One such condition is the common cardiomyopathy that occurs in patients receiving anthracyclines, such as doxorubicin, for cancer. Mukhopadhyay et al. observed that rimonabant markedly improved cardiac dysfunction and reduced doxorubicin-induced apoptosis in the myocardium [14]. They studied a short-term rodent model. Current approaches to ameliorate the risk for this condition are unsatisfactory. Perhaps a short-term course of rimonabant in patients requiring doxorubicin could be helpful. This possibility should be pursued (Fig. 1). After all, Bill Clinton did not deny THC use; he just claimed that he didn't inhale. Perhaps that saved him!

Respectfully, Friedrich C. Luft 


\section{References}

1. Howlett AC, Barth F, Bonner TI, Cabral G, Casellas P, Devane WA, Felder CC, Herkenham M, Mackie K, Martin BR et al (2002) International Union of Pharmacology. XXVII. Classification of cannabinoid receptors. Pharmacol Rev 54:161-202

2. Begg M, Pacher P, Bátkai S, Osei-Hyiaman D, Offertáler L, Mo FM, Liu J, Kunos G (2005) Evidence for novel cannabinoid receptors. Pharmacol Ther 106:133-145

3. Zimmer A, Zimmer AM, Hohmann AG, Herkenham M, Bonner TITI (1999) Increased mortality, hypoactivity, and hypoalgesia in cannabinoid CB1 receptor knockout mice. Proc Natl Acad Sci U S A 96:5780-5785

4. Pacher P, Mechoulam R (2011) Is lipid signaling through cannabinoid 2 receptors part of a protective system? Prog Lipid Res 50(2):193-211

5. Járai Z, Wagner JA, Varga K, Lake KD, Compton DR, Martin BR, Zimmer AM, Bonner TI, Buckley NE, Mezey E et al (1999) Cannabinoid-induced mesenteric vasodilation through an endothelial site distinct from $\mathrm{CB} 1$ or $\mathrm{CB} 2$ receptors. Proc Natl Acad Sci U S A 96:14136-14141

6. McHugh D, Tanner C, Mechoulam R, Pertwee RG, Ross RA (2008) Inhibition of human neutrophil chemotaxis by endogenous cannabinoids and phytocannabinoids: evidence for a site distinct from CB1 and CB2. Mol Pharmacol 73:441-450

7. Demuth DG, Molleman A (2006) Cannabinoid signalling. Life Sci 78:549-563
8. Fong TM, Heymsfield SB (2009) Cannabinoid-1 receptor inverse agonists: current understanding of mechanism of action and unanswered questions. Int J Obes (Lond) 33:947-955

9. Huestis MA et al (2001) Blockade of effects of smoked marijuana by the CB1-selective cannabinoid receptor antagonist SR141716. Arch Gen Psychiatry 58:322-328

10. Sslavic S, Lauer D, Sommerfeld M, Kemnitz UR, Grzesiak A, Trappiel M, Thöne-Reineke C, Baulmann J, Paulis L, Kappert K et al (2013) Cannabinoid receptor 1 inhibition improves cardiac function and remodeling after myocardial infarction and in experimental metabolic syndrome. J Mol Med. doi:10.1007/s00109-013-1034-0

11. Lim SY, Davidson SM, Yellon DM, Smith CCT (2009) The cannabinoid CB1 receptor antagonist, rimonabant protects against acute myocardial infarction. Basic Res Cardiol 104(6):781-792

12. Montecucco F, DiMarzo V (2012) At the heart of the matter: the endocannabinoid system in cardiovascular function and dysfunction. Trends Pharmacol Sci 33:331-340

13. Rajesh M, Mukhopadhyay P, Bátkai S, Patel V, Saito K, Matsumoto S, Kashiwaya Y, Horváth B, Mukhopadhyay B, Becker L et al (2010) Cannabidiol attenuates cardiac dysfunction, oxidative stress, fibrosis, and inflammatory and cell death signaling pathways in diabetic cardiomyopathy. J Am Coll Cardiol 56:2115-2125

14. Mukhopadhyay P, Bátkai S, Rajesh M, Czifra N, Harvey-White J, Haskó G, Zsengeller Z, Gerard NP, Liaudet L, Kunos G et al (2007) Pharmacological inhibition of CB1 cannabinoid receptor protects against doxorubicin-induced cardiotoxicity. J Am Coll Cardiol 50:528-536 